\title{
Clinical Results of Arthroscopic Rotator Cuff Repair with a Tape-Attached Anchor
}

\author{
Noriaki Shimada1, Jun'ichi Inoue ${ }^{2}$, Katsuhiko Shiraki' ${ }^{1}$, Kazuo Saita1 \\ ${ }^{1}$ Department of Orthopaedic Surgery, Saitama Medical Center, Saitama Medical University, Kawagoe, Japan \\ ${ }^{2}$ Department of Orthopaedic Surgery, Kamifukuoka General Hospital, Fujimino, Japan \\ Email: shima-50@live.jp
}

How to cite this paper: Shimada, N., Inoue, J., Shiraki, K. and Saita, K. (2018) Clinical Results of Arthroscopic Rotator Cuff Repair with a Tape-Attached Anchor. Open Journal of Orthopedics, 8, 249-256. https://doi.org/10.4236/ojo.2018.87028

Received: May 30, 2018

Accepted: June 30, 2018

Published: July 4, 2018

Copyright $\odot 2018$ by authors and Scientific Research Publishing Inc. This work is licensed under the Creative Commons Attribution International License (CC BY 4.0).

http://creativecommons.org/licenses/by/4.0/

\begin{abstract}
Objective: The purpose of this study was to evaluate the clinical results of arthroscopic rotator cuff repair with a tape-attached anchor: The HEALICOILRG with ULTRATAPE. We hypothesised that performing arthroscopic rotator cuff repair with tape-attached anchors would result in better clinical outcomes and reduce the retear rate compared with conventional suture anchors. Methods: We included 83 patients treated by arthroscopic rotator cuff repair with a minimum 1-year follow-up. We divided them into two groups: Use of the HEALICOILRG with ULTRATAPE (group T: 41 shoulders; mean patient age, 64.3 years) and use of the conventional suture anchor (group S: 42 shoulders; mean patient age, 68.9 years). We compared the University of California Los Angeles scores, Japanese Orthopaedic Association scores, range of motion, and retear rate between the two groups. The Student $t$ test and chi-square test were used in statistical analyses. Results: At the 1-year postoperative follow-up, both groups showed improvement in the average University of California Los Angeles score, Japanese Orthopaedic Association score, and range of motion, although no significant difference was found in the retear rate between the two groups. Conclusions: Results of arthroscopic rotator cuff repair with the HEALICOILRG with ULTRATAPE were generally good. However, our results could not demonstrate efficacy of the HEALICOILRG with ULTRATAPE. Further detailed studies are needed to determine its treatment result.
\end{abstract}

\section{Keywords}

Arthroscopic Rotator Cuff Repair, Transosseous-Equivalent Technique, Suture Anchor, Tape-Attached Anchor, Retear

\section{Introduction}

Arthroscopic rotator cuff repair (ARCR) has an established place in treating ro- 
tator cuff tears. Many studies have reported methods, results, and availabilities of ARCR [1]-[9]. The transosseous-equivalent (TOE) technique has been popularised in many methods of ARCR because it can hold down the cuff widely [5]-[12]. Strong tape, which is wider than a strong suture, was developed, so we became able to use a tape-attached anchor. The efficacy of the tape compared with the suture has been demonstrated in some biomechanical studies [13] [14] [15] [16]. However, the efficacy of the tape has not been clarified in the clinical setting, as there are few reports about its clinical result [16].

This study aimed to evaluate the clinical results of ARCR with tape-attached anchors. We hypothesised that performing ARCR with tape-attached anchors would result in better clinical outcomes and reduce the retear rate compared with conventional suture anchors.

\section{Materials and Methods}

Our inclusion criteria were patients with a full-thickness rotator cuff tear who underwent ARCR with the TOE technique between December 2015 and December 2016; those who received a tape-attached anchor, the HEALICOILRG with ULTRATAPE (HCUT; Smith \& Nephew, Memphis, TN, USA) as the medial anchor (group $\mathrm{T}$ ) or a conventional suture anchor as the medial anchor (group S); and those who had a minimum 1-year follow-up. We excluded patients with a massive rotator cuff tear and those who underwent partial repair, fascia lata patch repair, and revision surgery. We used a conventional suture anchor until June 2016 and then used HCUT since July 2016. Informed consent was obtained from all the patients who participated in this study.

We compared the University of California Los Angeles (UCLA) scores, Japanese Orthopaedic Association (JOA) scores, range of motion (ROM), and retear rate between the two groups. We also compared the UCLA and JOA scores and ROM between preoperation and the 1-year postoperative follow-up. Sugaya's classification was used to evaluate the cuff integrity by magnetic resonance imaging 6 months postoperatively, with types IV and V classified as rotator cuff retears.

All surgeries were performed under general anaesthesia and an interscalene block with the patient in the beach-chair position. After the arthroscopic examinations and assessment of the rotator cuff tear, all patients underwent synovial tissue removal to mobilise the rotator cuff and acromioplasty before repair rotator cuff. All surgeries were performed using the TOE technique. We used suture anchors in the medial row and knotless anchors in the lateral row with the TOE technique.

In group T, we placed 1 to 2 HCUTs as medial anchors at the articular cartilage margin. These tapes and sutures of the anchors were passed through the rotator cuff using a suture hook, and we added several independent sutures as needed. We used 1 to 2 lateral knotless anchors (Swive Lock; Arthrex, Naples, FL, USA) to fix these tapes and sutures at the lateral cortex below the greater tu- 
berosity. Finally, medial row knots were created with the remaining sutures. In group $\mathrm{S}$, we performed almost the same method as in group $\mathrm{T}$, but we used conventional anchors as the medial anchors instead of HCUT.

Postoperatively, the operative arm was supported for 6 weeks in a sling with a small abduction pillow. At 3 weeks, passive ROM exercises were started. At 6 weeks, active ROM exercises were begun. Shoulder-strengthening exercises were performed at 9 to 12 weeks.

Statistical analysis was performed by using IBM SPSS statistics 24 (IBM Corp., Armonk, NY). Differences between the two groups were analysed using the Student $\mathrm{t}$ test and chi-square test. For all analyses, a $P$-value $<0.05$ was considered significant.

\section{Results}

The study included 83 patients ( 41 patients in group $\mathrm{T}$ and 42 patients in group S). Patient demographic characteristics are presented in Table 1. Group T consisted of 23 men and 18 women, with a mean age of $65.3 \pm 8.9$ years. Group S consisted of 21 men and 21 women, with a mean age of $68.9 \pm 5.6$ years. In group $\mathrm{T}$, the right shoulder was operated on in 26 patients and the left in 15 . In group $\mathrm{S}$, the right shoulder was operated on in 24 patients and the left in 18 . The average durations of symptoms were $6.1 \pm 3.8$ months in group $\mathrm{T}$ and $5.9 \pm 3.6$ in group S. There were 19 patients with a small tear, 12 with a medium tear, and 10 with a large tear in group $\mathrm{T}$. In group S, there were 17 patients with a small tear, 13 with a medium tear, and 12 with a large tear.

There were no significant differences in age, sex, the affected shoulder, duration of symptoms, and tear size between the groups.

Both groups showed postoperative improvement in the UCLA score, JOA score, and ROM. Between the two groups, there were no significant differences preoperatively or at the 1-year postoperative follow-up, and no significant differences in the changes from before the operation to the 1-year postoperative follow-up (Table 2).

Both groups showed postoperative improvement in the range of anterior elevation and external rotation. There were no significant differences preoperatively or at the 1-year postoperative follow-up and no significant differences in the changes from before the operation to the 1-year postoperative follow-up between the two groups (Table 3).

There were also no significant differences in the retear rate between the two groups (Table 4).

\section{Discussion}

With the evolution of the arthroscopic technique, ARCR is now the standard treatment for rotator cuff tears. Surgeons have sought to improve the clinical result of ARCR with the development of instruments and technical advancements. Many studies have reported a good clinical result of ARCR with the TOE 
Table 1. Patient demographic data.

\begin{tabular}{cccc}
\hline & Group T $(\mathrm{n}=41)$ & Group S $(\mathrm{n}=42)$ & $P$-value \\
\hline Age, years & $65.3 \pm 8.9$ & $68.9 \pm 5.6$ & 0.087 \\
Sex (male/female) & $23 / 18$ & $21 / 21$ & 0.621 \\
Affected shoulder (right/left) & $26 / 15$ & $24 / 18$ & 0.667 \\
Duration of symptoms, months & $6.1 \pm 3.8$ & $5.9 \pm 3.6$ & 0.552 \\
Tear size & $19 / 12 / 10$ & $17 / 13 / 12$ & 0.323 \\
(small/medium/large) & & & \\
\hline
\end{tabular}

Group T, use of the HEALICOILRG with ULTRATAPE; group S, use of the conventional suture anchor.

Table 2. Comparison of the clinical outcome between group $\mathrm{T}$ and group $\mathrm{S}$.

\begin{tabular}{|c|c|c|c|c|c|}
\hline & \multicolumn{2}{|c|}{ Preoperatively } & \multicolumn{2}{|c|}{ Postoperatively } & \multirow{2}{*}{$P$-value } \\
\hline & Mean \pm SD & $P$-value & Mean \pm SD & $P$-value & \\
\hline \multicolumn{6}{|l|}{ UCLA score } \\
\hline Group T & $13.4 \pm 4.4$ & \multirow{2}{*}{0.723} & $32.7 \pm 3.9$ & \multirow{2}{*}{0.668} & $<0.001$ \\
\hline Group S & $13.8 \pm 4.0$ & & $33.3 \pm 3.6$ & & $<0.001$ \\
\hline \multicolumn{6}{|l|}{ JOA score } \\
\hline Group T & $63.8 \pm 7.4$ & \multirow{2}{*}{0.656} & $88.4 \pm 8.2$ & \multirow{2}{*}{0.523} & $<0.001$ \\
\hline Group S & $62.5 \pm 7.4$ & & $87.4 \pm 8.1$ & & $<0.001$ \\
\hline
\end{tabular}

Group T, use of the HEALICOILRG with ULTRATAPE; group S, use of the conventional suture anchor SD, standard deviation; JOA, Japanese Orthopaedic Association; UCLA, University of California Los Angeles.

Table 3. Comparison of range of motion between group $\mathrm{T}$ and group $\mathrm{S}$.

\begin{tabular}{|c|c|c|c|c|c|}
\hline & \multicolumn{2}{|c|}{ Preoperatively } & \multicolumn{2}{|c|}{ Postoperatively } & \multirow{2}{*}{$P$-value } \\
\hline & Mean \pm SD & P-value & Mean \pm SD & $P$-value & \\
\hline \multicolumn{6}{|c|}{ Anterior elevation } \\
\hline Group T & $118.2 \pm 26$ & \multirow{2}{*}{0.422} & $148.3 \pm 13$ & \multirow{2}{*}{0.536} & $<0.001$ \\
\hline Group S & $113.8 \pm 25$ & & $144.8 \pm 18$ & & $<0.001$ \\
\hline \multicolumn{6}{|c|}{ External rotation } \\
\hline Group T & $37.8 \pm 12$ & \multirow{2}{*}{0.512} & $51.8 \pm 6.8$ & \multirow{2}{*}{0.861} & $<0.001$ \\
\hline Group S & $40.2 \pm 20$ & & $52.7 \pm 12$ & & $<0.001$ \\
\hline
\end{tabular}

Group T, use of the HEALICOILRG with ULTRATAPE; group S, use of the conventional suture anchor SD, standard deviation.

Table 4. Comparison of the retear rate between group $\mathrm{T}$ and group $\mathrm{S}$.

\begin{tabular}{cccc}
\hline & Group T $(\mathrm{n}=41)$ & Group S $(\mathrm{n}=42)$ & $P$-value \\
\hline Retear & 8 & 7 & \\
Intact & 33 & 35 & 0.782 \\
Retear rate $(\%)$ & 19.5 & 16.7 &
\end{tabular}

Group T, use of the HEALICOILRG with ULTRATAPE; group S, use of the conventional suture anchor.

technique [10] [11] [12] [17] [18] [19] [20] [21]. Recently, a tape material was developed as a substitution for the suture of the anchor. We began using the 
tape-attached anchor HCUT instead of the conventional suture anchor. HCUT has one strong tape and one strong suture; therefore, stable restoration is expected. Some biomechanical studies have reported that the tape was superior to the suture in construct strength [13] [14] [15] [16]. Bisson et al. reported that the tape was three times as stiff as the suture, and it failed at three times the loads of the suture [13]. Carli et al. reported that the tape is biomechanically stronger than a suture, and the tape structure does not have a weak point [14]. Barber et al. reported that repair using the tape and suture had a greater mean ultimate failure load than repair using the suture only in their biomechanical study [15]. Liu et al. reported that the tape provided a three-fold increase in the footprint contact pressure and a one and a half-fold increase in construct strength compared with the suture in biomechanical study [16]. However, there have been very few reports on the clinical result of using the tape material with ARCR, so its clinical usefulness has not been clarified yet.

We used HCUT to improve clinical results, in particular to reduceretears. We think that the tape material can provide better restoration by the fixing the rotator cuff more widely, and it can provide good results, as reported by some biomechanical studies [13] [14] [15] [16]. In fact, significant improvements were seen in both the clinical score and ROM with ARCR using HCUT. However, there were no clinical differences in the clinical scores, ROM, and retear rate between the ARCR using HCUT and the conventional method. By using HCUT, we could not achieve a better clinical result or reduce the retear rate. The efficacy of the tape, which has been demonstrated in biomechanical studies [13] [14] [15] [16], was not effective based on the clinical outcomes in our study. Liu et al. reported an advantage of tape in their biomechanical study, but they reported that this advantage did not translate to better clinical outcomes; there was no clinical difference in the retear rate between the tape and suture groups [16]. Deranlot et al. reported the abrasive properties of some different sutures and tape materials [22]. They suspected that the tape material is less irritating inside the tendon and would decrease the abrasive effects, but results of their biomechanical study did not support their hypothesis; the abrasive effects of the tape material were increased compared with some sutures. We thought that the tape material disperses the pressure and is less invasive because it holds the rotator cuff widely. It is noteworthy that Deranlot et al.'s study stated that the tape was rather invasive to the rotator cuff. We need to further investigate the effect and invasion of tape material on the rotator cuff. We also must consider the initial strength improvement and the abrasive properties of the tape. In addition, we need to examine the effect of tape and the suture on the base bed of the humerus, haemodynamics of repaired rotator cuffs, and durability of the sutures and tapes. In order to improve the clinical result of ARCR, we have to consider many more issues. In any case, the improvement of initial strength of the tape confirmed by biomechanical studies was not directly related to improvement of the clinical results in our study. Our study suggested that there is something more important than initial strength in the healing rotator cuff. 
Our study has limitations. The study sample was relatively small and the follow-up period was short, which might be the reasons why there was no significant difference in the clinical results and retear rates between the two groups.

\section{Conclusion}

Our results could not demonstrate the efficacy of HCUT. Further detailed studies are needed to determine its treatment result.

\section{Acknowledgements}

We would like to thank Editage (http://www.editage.jp/) for English editing.

\section{Conflicts of Interest Statement}

The authors have no conflicts relevant to this article.

\section{Funding/Support Statement}

The authors received no financial support for the research, authorship, and/or publication of this article.

\section{References}

[1] Cole, B.J., McCarty 3rd, L.P., Kang, R.W., et al. (2007) Arthroscopic Rotator Cuff Repair: Prospective Functional Outcome and Repair Integrity at Minimum 2-Year Follow-Up. Journal of Shoulder and Elbow Surgery, 16, 579-585. https://doi.org/10.1016/j.jse.2006.12.011

[2] Vaishnav, S. and Millett, P.J. (2010) Arthroscopic Rotator Cuff Repair: Scientific Rationale, Surgical Technique, and Early Clinical and Functional Results of a Knotless Self-Reinforcing Double-Row Rotator Cuff Repair System. Journal of Shoulder and Elbow Surgery, 19, 83-90. https://doi.org/10.1016/j.jse.2009.12.012

[3] DeFranco, M.J., Bershadsky, B., Ciccone, J., Yum, J.K. and Iannotti, J.P. (2007) Functional Outcome of Arthroscopic Rotator Cuff Repairs: A Correlation of Anatomic and Clinical Results. Journal of Shoulder and Elbow Surgery, 16, 759-765. https://doi.org/10.1016/j.jse.2007.03.020

[4] Liu, J., Fan, L., Zhu, Y., Yu, H., Xu, T. and Li, G. (2017) Comparison of Clinical Outcomes in All-Arthroscopic versus Mini-Open Repair of Rotator Cuff Tears: A Randomized Clinical Trial. Medicine (Baltimore), 96, e6322. https://doi.org/10.1097/MD.0000000000006322

[5] Hughes, J.D., Hughes, J.L., Bartley, J.H., Hamilton, W.P. and Brennan, K.L. (2017) Infection Rates in Arthroscopic versus Open Rotator Cuff Repair. Orthopaedic Journal of Sports Medicine, 5, 2325967117715416. https://doi.org/10.1177/2325967117715416

[6] Kang, L., Henn, R.F., Tashjian, R.Z. and Green, A. (2007) Early Outcome of Arthroscopic Rotator Cuff Repair: A Matched Comparison with Mini-Open Rotator Cuff Repair. Arthroscopy, 23, 573-582, 582.e1-e2.

[7] Jensen, A.R., Cha, P.S., Devana, S.K., et al. (2017) Evaluation of the Trends, Concomitant Procedures, and Complications with Open and Arthroscopic Rotator Cuff Repairs in the Medicare Population. Orthopaedic Journal of Sports Medicine, 5, 2325967117731310. https://doi.org/10.1177/2325967117731310 
[8] Baker, D.K., Perez, J.L., Watson, S.L., et al. (2017) Arthroscopic versus Open Rotator Cuff Repair: Which Has a Better Complication and 30-Day Readmission Profile? Arthroscopy, 33, 1764-1769. https://doi.org/10.1016/j.arthro.2017.04.019

[9] Lucena, T.R., Lam, P.H., Millar, N.L. and Murrell, G.A. (2015) The Temporal Outcomes of Open versus Arthroscopic Knotted and Knotless Rotator Cuff Repair over 5 Years. Shoulder Elbow, 7, 244-255. https://doi.org/10.1177/1758573215581775

[10] Park, M.C., ElAttrache, N.S., Tibone, J.E., Ahmad, C.S., Jun, B.J. and Lee, T.Q. (2007) Part I: Footprint Contact Characteristics for a Transosseous-Equivalent Rotator Cuff Repair Technique Compared with a Double-Row Repair Technique. Journal of Shoulder and Elbow Surgery, 16, 461-468. https://doi.org/10.1016/j.jse.2006.09.010

[11] Neyton, L., Godenèche, A., Nové-Josserand, L., Carrillon, Y., Cléchet, J. and Hardy, M.B. (2013) Arthroscopic Suture-Bridge Repair for Small to Medium Size Supraspinatus Tear: Healing Rate and Retear Pattern. Arthroscopy, 29, 10-17. https://doi.org/10.1016/j.arthro.2012.06.020

[12] Sethi, P.M., Noonan, B.C., Cunningham, J., Shreck, E. and Miller, S. (2010) Repair Results of 2-Tendon Rotator Cuff Tears Utilizing the Transosseous Equivalent Technique. Journal of Shoulder and Elbow Surgery, 19, 1210-1217. https://doi.org/10.1016/j.jse.2010.03.018

[13] Bisson, L.J. and Manohar, L.M. (2010) A Biomechanical Comparison of the Pullout Strength of No. 2 FiberWire Suture and 2-mm FiberWire Tape in Bovine Rotator Cuff Tendons. Arthroscopy, 26, 1463-1468. https://doi.org/10.1016/j.arthro.2010.04.075

[14] De Carli, A.D., Lanzetti, R.M. and Monaco, E. (2012) The Failure Mode of Two Reabsorbable Fixation Systems: Swivelock with Fibertape versus Bio-Corkscrew with Fiberwire in Bovine Rotator Cuff. Journal of Orthopaedic Science, 17, 789-795. https://doi.org/10.1007/s00776-012-0275-Z

[15] Barber, F.A. and Drew, O.R. (2012) A Biomechanical Comparison of Tendon-Bone Interface Motion and Cyclic Loading between Single-Row, Triple-Loaded Cuff Repairs and Double-Row, Suture-Tape Cuff Repairs Using Biocomposite Anchors. Arthroscopy, 28, 1197-1205. https://doi.org/10.1016/j.arthro.2012.02.015

[16] Liu, R.W., Lam, P.H., Shepherd, H.M. and Murrell, G.A.C. (2017) Tape versus Suture in Arthroscopic Rotator Cuff Repair. Orthopaedic Journal of Sports Medicine, 5, Article ID: 2325967117701212. https://doi.org/10.1177/2325967117701212

[17] Gartsman, G.M., Drake, G., Edwards, T.B., et al. (2013) Ultrasound Evaluation of Arthroscopic Full-Thickness Supraspinatus Rotator Cuff Repair: Single-Row versus Double-Row Suture Bridge (Transosseous Equivalent) Fixation. Results of a Prospective, Randomized Study. Journal of Shoulder and Elbow Surgery, 22, 1480-1487. https://doi.org/10.1016/j.jse.2013.06.020

[18] Lee, K.W., Seo, D.W., Bae, K.W. and Choy, W.S. (2013) Clinical and Radiological Evaluation after Arthroscopic Rotator Cuff Repair Using Suture Bridge Technique. Clinics in Orthopedic Surgery, 5, 306-313. https://doi.org/10.4055/cios.2013.5.4.306

[19] Miyazaki, A.N., Santos, P.D., Sella, G.D., Checchia, C.S., Salata, T.R. and Checchia, S.L. (2017) Evaluation of the Functional Results after Rotator Cuff Arthroscopic Repair with the Suture Bridge Technique. Revista Brasileira De Ortopedia, 52, 164-168. https://doi.org/10.1016/j.rbo.2016.05.002

[20] Cho, N.S., Yi, J.W., Lee, B.G. and Rhee, Y.G. (2010) RetearPatterns after Arthroscopic Rotator Cuff Repair: Single-Row versus Suture Bridge Technique. The American Journal of Sports Medicine, 38, 664-671. 
https://doi.org/10.1177/0363546509350081

[21] Abdelshahed, M., Mahure, S.A., Kaplan, D.J., et al. (2016) Arthroscopic Rotator Cuff Repair: Double-Row Transosseous Equivalent Suture Bridge Technique. Arthroscopy Techniques, 5, e1297-e1304. https://doi.org/10.1016/j.eats.2016.07.022

[22] Deranlot, J., Maurel, N., Diop, A., Pratlong, N., Roche, L., Tiemtore, R. and Nourissat, G. (2014) Abrasive Properties of Braided Polyblend Sutures in Cuff Tendon Repair: An in Vitro Biomechanical Study Exploring Regular and Tape Sutures. Arthroscopy, 30, 1569-1573. https://doi.org/10.1016/j.arthro.2014.06.018 